\title{
Topological optomechanically induced transparency
}

\author{
FARZAd ZANGeneh-NejAD, ROMAIn FLeURY* \\ Labratory of Wave Engineering, Swiss Federal Institute of Technology in Lausanne (EPFL), 1015, Lausanne, Switzerland \\ *Corresponding author: romain.fleury@epfl.ch
}

Received XX Month XXXX; revised XX Month, XXXX; accepted XX Month XXXX; posted XX Month XXXX (Doc. ID XXXXX); published XX Month XXXX

\begin{abstract}
The interaction of optical and mechanical degrees of freedom can lead to several interesting effects. A prominent example is the phenomenon of optomechanically induced transparency (OMIT), in which mechanical movements induce a narrow transparency window in the spectrum of an optical mode. In this Letter, we demonstrate the relevance of optomechanical topological insulators for achieving OMIT. More specifically, we show that the strong interaction between optical and mechanical edge modes of a one-dimensional topological optomechanical crystal can render the system transparent within a very narrow frequency range. Since the topology of a system cannot be changed by slight to moderate levels of disorder, the achieved transparency is robust against geometrical perturbations. This is in sharp contrast to trivial OMIT which has a strong dependency on the geometry of the optomechanical system. Our findings hold promises for a wide range of applications such as filtering, signal processing, and slow-light devices.
\end{abstract}

Electromagnetic induced transparency (EIT) is a phenomenon arising from the constructive and destructive interferences between two different optical modes, leading to the cancellation of the light absorption within a very small spectral range called transparency window [1-3]. In the transparency window, the absorption spectrum varies very rapidly. According to the Kramers-Kronig [4] relations, such an abrupt change of the absorption coefficient comes with a rapid change of the refractive index. This gives rise to a significant reduction in the group velocity of light, enabling exciting applications such as optical buffering $[5,6]$, photonic quantum memory $[7,8]$, optical rotation sensing $[9,10]$, and data synchronization $[11,12]$.

So far, various approaches have been proposed to achieve electromagnetic induced transparency. One common and established method, known as coupled resonator induced transparency (CRIT) technique [13-17], relies on the interaction between a pair of optical resonators (with the resonance frequency of $\omega_{0}$, for example). The destructive interference between the resonators splits the spectrum of the modes into two separate peaks (one before and one after $\omega_{0}$ ), opening a narrow transparent region around $\omega_{0}$. Despite its simple principle, the CRIT technique is associated with several drawbacks. In particular, the coupled resonance induced transparency is very fragile to disorder, as the characteristics of the involved resonances are exclusively enforced by the geometry. This renders controlling CRIT extremely challenging.

Another conceptually distinct route to achieve electromagnetic induced transparency is to leverage the interaction of an optical mode with mechanical motions. Under certain conditions, the strong interaction between these two degrees of freedom can result in a Fano-like resonance $[18,19]$, characterized by an ultra-sharp peak-and-dip spectrum. In the frequency range where the dip of the Fano resonance occurs, the optomechanical system becomes completely transparent. Compared to CRIT, this type of transparency, known as optomechanically induced transparency (OMIT) [20-23], has several advantageous features. Firstly, OMIT can be tuned very easily by controlling the interaction between mechanical and optical degrees of freedom. In fact, any parameter that affects the mechanical properties of the opto-mechanical system (such as the temperature or pressure of the ambient) can be used as a tool to manipulate the characteristics of the EIT at will. Additionally, OMIT is based on only one single opto-mechanical element, representing significant miniaturization compared to the structures based on the CRIT technique (that are composed of two independent components). Yet, similar to CRIT, OMIT is sensitive to imperfections coming from fabrication tolerances, or the presence of defects, as the characteristics of the optical and mechanical parts still rely on maintaining a pristine geometry of the opto-mechanical structure.

In a seemingly unrelated field of science, artificial insulating materials with non-trivial topological band structures, known as topological insulators (TIs) [24-28], have attracted a lot of attention for their unprecedented robustness against geometrical variations. On their edges, finite-size TIs support resonant modes, whose existence is only related to the topological characteristics of the bulk system. Destroying their presence can only be achieved through drastic global modifications that close the insulating bandgaps, implying that they are robust to moderate levels of geometrical modifications [25]. This leads to strong resilience against certain types of disorder. Such an appealing feature has enabled the development of a large variety of disorder-immune 




Fig. 1. Concept of topological optomechanically induced transparency, Top: Consider a trivial optomechanical system, supporting an optical and a mechanical resonance mode. The interaction between the two resonators opens a transparency window in the absorption spectrum of the system. Bottom: When some disorder is introduced to the system, the characteristics of the two involved resonances are not significantly changed.

structures in various fields of interest including photonic [28,30], phononics $[31,32]$ and mechanics $[33,34]$.

In this Letter, we demonstrate the possibility of achieving OMIT by engineering the topology of an optomechanical system. In particular, we show that the coupling between the optical and mechanical edge modes of a one-dimensional optomechanical crystal can give rise to a topological resonance [35], featuring a very small transparency window. Advantageously, the obtained OMIT is not related to the geometry of the optomechanical crystal, since the optical and mechanical resonances are due to topological nature of the system. The ultra-sharp spectrum of the obtained topological OMIT holds great promises for a large variety of applications such as sensing, signal processing, and optical data storage.

The working principle of a regular (trivial) optomechanical system is based on the coupling between an optical and a mechanical mode. The strong interaction between these modes creates an absorption spectrum with a very small transparency window. Since the system is topologically trivial, the characteristics of the involved resonances are mainly determined by the geometry of the opto-mechanical system. As such, the corresponding OMIT is extremely sensitive to imperfections. In order to mitigate these harmful effects, we propose to achieve OMIT based on the strong interaction between an optical and a mode whose existence is linked not to a definite geometry but to the topology of an optomechanical crystal (see Figure 1a). Since the topology of a system is preserved under continuous deformation of the band structure, slight to moderate geometrical perturbations may not affect the spectral characteristics of the optical and mechanical resonances. As a result, as opposed to the trivial case, the corresponding OMIT is robust (Fig. 1b).

In order to provide a concrete example of topological OMIT, we consider a one-dimensional photonic crystal, based on the unit-cell shown in Fig. 2a (top panel). The unit cell consists of a thin silicon wafer perforated with a rectangular hole. The parameters $a, b, W$, $h$ are chosen as $a=0.36 \mu m, b=0.99 \mu m, W=1.4 \mu m, h=$ $0.19 \mu \mathrm{m}$ respectively. The thickness of the silicon wafer is
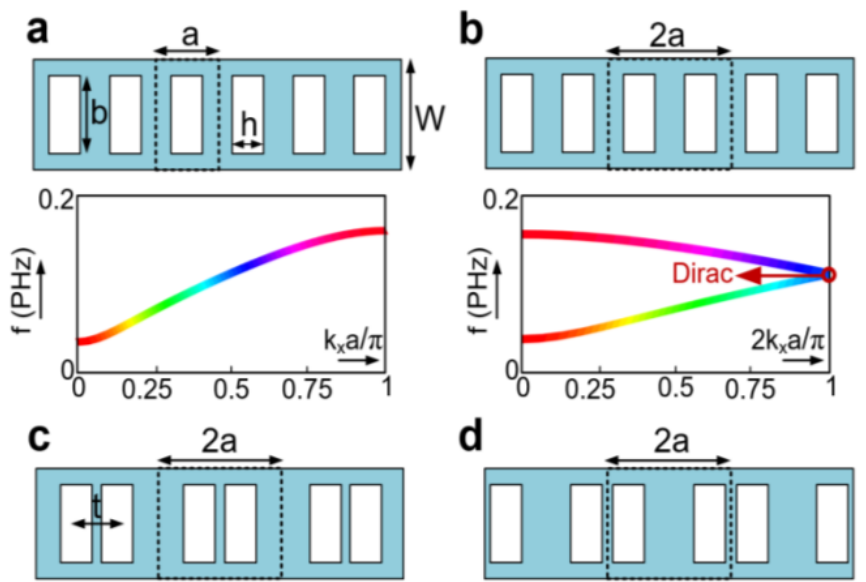

d
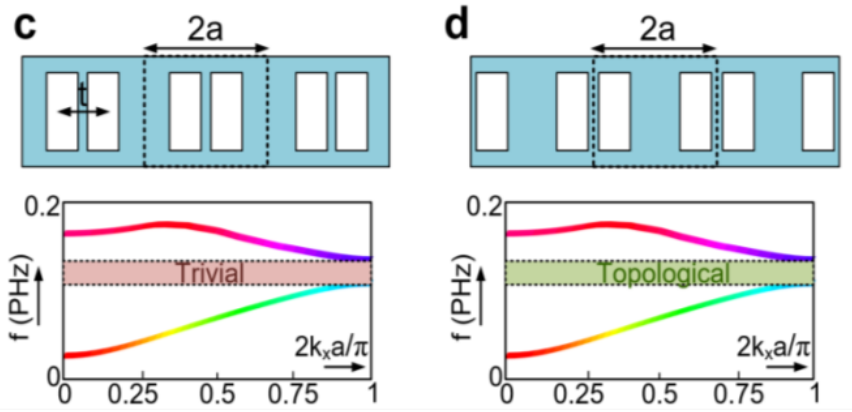

Fig. 2. Proposed topological structure for achieving OMIT, a, (Top) an opto-mechanical crystal consisting of a thin silicon wafer (blue area) perforated with rectangular silicon holes. (Bottom) Dispersion band of the fundamental optical mode of the structure. $b$, Band structures of the extended unit-cell of the crystal. The dispersion diagram exhibits point degeneracy at the edges of the BZ due to the band folding effect. c, The distance between the holes inside the extended unit-cell of the crystal is decreased so as to open an insulating band gap. The band gap is characterized with a trivial topological order. $d$, Same as c except that the distance between the holes in the extended unit cell is increased, leading to a topologically non-trivial insulating band gap.

chosen as $t=0.22 \mu \mathrm{m}$. Fig. 2a (bottom panel) represents the dispersion of the fundamental optical mode of the photonic crystal, obtained by performing numerical simulations based on a standard finite element method (Comsol Multi-physics, electromagnetic (RF) module).

In order to induce non-trivial topological features in such system, we double the size of the unit cell to fold the band, and investigate the dispersion properties of the super-cell of the crystal. Fig. $2 b$ shows the corresponding dispersion diagram. The two dispersion bands now cross each other at point degeneracies located the edges of the (new) Brillouin zone. Such degeneracies represent a particularly relevant starting points for engineering the topology of the system, since lifting a band degeneracy may be accompanied by the exchange of topological charges. This can be achieved by introducing symmetry-lowering mechanisms. The degeneracy in the band structure of Fig. $2 \mathrm{~b}$ is related to the sub-lattice symmetry of the super-cell, namely the reflection symmetry with respect to the position $x=a / 2$. One way to break this symmetry is to reduce the distance between the holes inside the super-cell (Fig. 2c). The bottom panel of Fig. 2c illustrates the corresponding band structure. As expected, the twofold degeneracies have been split, leading to an insulating band gap. This insulting band gap is characterized by a zero topological invariant [24]. 
a

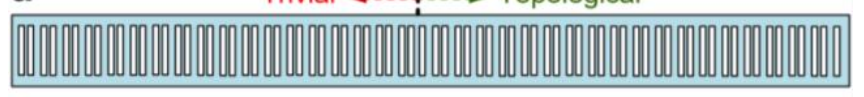

b

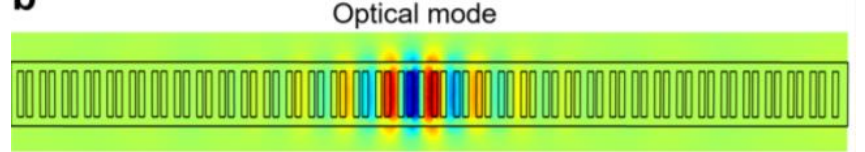

C

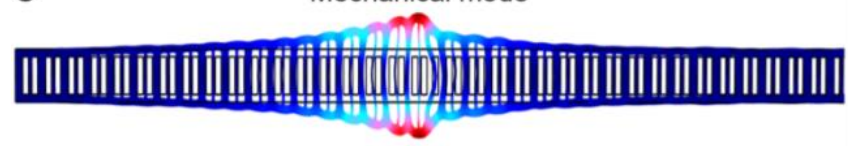

d
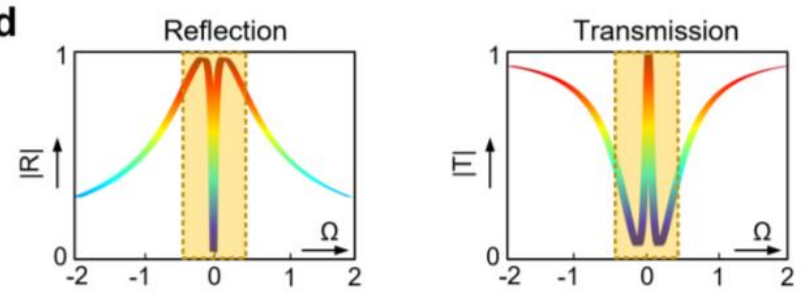

Fig. 3. Numerical Simulations of a topological OMIT, a, we connect a finite piece of the trivial crystal to the topological one, so as to achieve photonic and phononic topological edge modes $b$, Profile of the corresponding optical topological edge mode at the interface between the trivial and topological crystals. c, Profile of the corresponding mechanical topological edge mode. $\mathrm{d}$, (Left) Corresponding reflection spectrum of the system, exhibiting an ultra-sharp spectral line shape caused by the inference between the optical and mechanical edge modes. (Right) Corresponding transmission spectrum, featuring a very narrow transparency window (yellow region).

The alternative strategy to break the sub-lattice symmetry of the super-cell resides in increasing the distance between the holes inside the super-cell. Fig. $2 \mathrm{~d}$ represents the corresponding band structure. Like the previous case, the crystal under investigation exhibits an insulating band gap, looking similar to that of Fig. 2c. From a topological point of view, however, this insulating band gap is different from the previous one. In particular, the latter insulating band gap is characterized by a non-zero topological order, as opposed to the former case in which the topological invariant was zero.

The most striking feature of systems with a non-trivial topology is that they support edge modes when they form a boundary with a trivial structure. In order to realize photonic edge modes, we connect a finite piece of the non-trivial crystal to the trivial one (Fig. 3a). We then perform eigen frequency simulation to find the associated topological edge mode. Inset of Figure $3 \mathrm{~b}$ illustrates the profile of the corresponding optical edge mode. It is observed that the edge mode is localized at the interface between the trivial and topological crystals.

On top of these photonic considerations, the structure shown in Fig. 3a can also be considered as a topological phononic system, supporting a "mechanical" topological edge mode at the interface between the two crystals with opposite hopping deformation. Shown in Fig. $3 c$ is the profile of the mechanical topological edge mode. Like its optical counterpart, the mechanical edge mode is confined to the phase transition interface, leading to a strong a

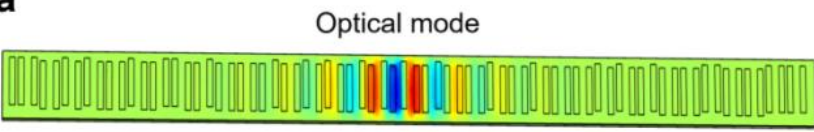

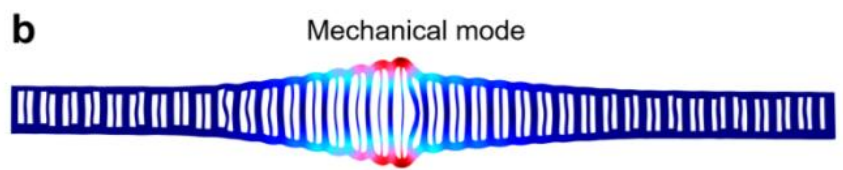

C
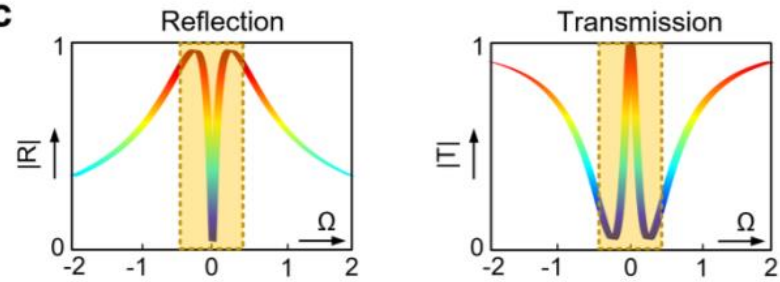

Fig.4. Robustness of topological optomechanical induced transparency, we introduce some disorder to the proposed topological optomechanical crystal by randomly changing the position of the rectangular holes, a, Profile of the corresponding topological optical edge mode. Thanks to the topological properties of the system, the edge mode is not affected by the disorder. c, Same as panel a but for the topological mechanical edge mode of the system, d, Corresponding reflection (left) and transmission (right) spectrum of the system.
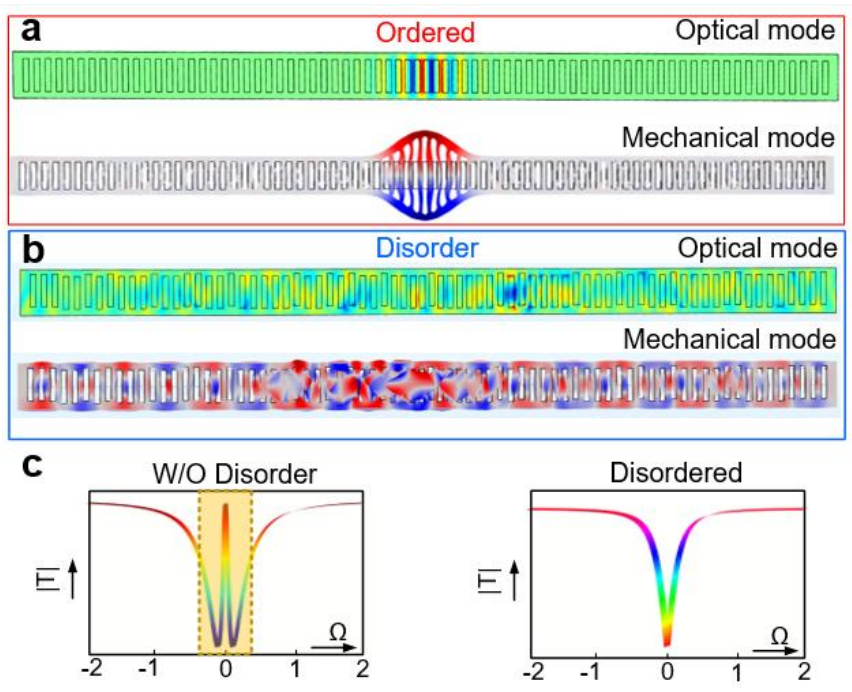

Fig. 5. Sensitivity of trivial optomechanical induced transparency, we introduce some disorder to the proposed topological optomechanical crystal by putting a defect inside a 1D crystal, a, Profile of the corresponding optical and mechanical mode. c, Same as panel a, but with the same disorder as in Fig. 4, c, Corresponding transmission spectra with (Right) and without (Left) disorder.

optomechanical interaction. By tuning the geometry of the structure, the interaction of the optical and mechanical edge modes is maximized. The coupling between the optical and mechanical topological degrees of freedom can be characterized with a parameter known as coupling length, defined as [22] 
$L_{c}=\left[\frac{\int\left[\Delta \varepsilon\left|E_{\|}\right|^{2}-\Delta \varepsilon^{-1}\left|\varepsilon E_{n}\right|^{2}\right](\boldsymbol{Q} . \hat{n}) d s}{\int \varepsilon|E|^{2} d V}\right]^{-1}$

in which $E_{\|}$and $E_{n}$ are the tangential and perpendicular parts of the optical field respectively, $Q$ is the unit-less displacement of the mechanical mode, $\Delta \varepsilon=\varepsilon_{S i}-\varepsilon_{\text {air }}$ and $\Delta \varepsilon^{-1}=\varepsilon_{S i}^{-1}-\varepsilon_{\text {air }}^{-1}$. By calculating the associated integrals in Eq. 1, the coupling length between the topological optical and mechanical edge modes was obtained as $L_{c}=2.01 \mu \mathrm{m}$. Once the coupling length between the optical and mechanical parts is found, the reflection and transmission spectrum of the optomechanical system can be calculated by using the following formulas [22]

$$
R=\frac{-\gamma_{\text {ext }} / 2}{i\left(\omega_{m}-\Delta\right)+\kappa / 2+\frac{|G|^{2}}{i\left(\omega_{m}-\Delta\right)+\gamma_{i} / 2}} ; T=1+R
$$

in which $\gamma_{\text {ext }}$ is the loss rate associated with the coupling to the excitation, $\omega_{m}$ is the frequency of the mechanical mode, $\gamma_{i}$ is the intrinsic damping rate of the mechanical mode, $\kappa$ is the optical decay rate, and $\Delta$ is the pump detuning from the optical cavity. $G=$ $L_{c}^{-1} \omega_{0} \sqrt{\hbar / 4 \pi m_{e f f} f_{m}}$ is the optomechanical coupling rate. Based on Eqs. 2 and 3, the corresponding reflection and transmission spectrum are calculated as a function of $\Omega=\left(\Delta-\omega_{m}\right) / \kappa$, and plotted in Figures $3 \mathrm{~d}$ and e, respectively. As it is observed, a very small transparency window is opened in the spectrum of the system due to the interference between the optical and mechanical edge modes. Within the transparency window, the transmission coefficient of the structure reaches unity, whereas the associated reflection coefficient drops to zero. Therefore, in this frequency range, the system is transparent.

The fact that topology of a system cannot be changed by slight to moderate geometrical modifications implies that the obtained topological OMIT should exhibit some sort of immunity against disorder. In order to verify this predication, we introduce some disorder to the system by randomly moving the positions of the rectangular holes, both horizontally and vertically, of the optomechanical crystal. Fig. 4a and $b$ illustrate the mode profiles of the corresponding optical and mechanical edge modes. As observed, neither the optical nor the mechanical edge mode is largely affected by the disorder. This is because the presence of both of these edge modes at the center of the band gap is guaranteed by the difference between the topological invariants of the trivial and non-trivial crystals [24]. Disorder, however, can change the coupling length between the optical and mechanical modes. This modifies a little bit the spectral line shape of the OMIT, as seen in the inset of Fig. 4c. This residual sensitivity to environment can be used in sensing applications to track the changes of environmental parameters. Nevertheless, the absence of frequency shifts and occurrence of parasitic peaks in the lineshape is a direct consequence of the topological nature of the modes, in stark constrast with the extreme sensitivity of the traditional OMIT mechanism. This extreme sensitivity is demonstrated in Fig. 5, Here, the coupling between the optical and mechanical defect modes (Fig. 5a)of a 1D crystal is used to induce OMIT (Fig. 5c, Left). When we add the same disorder as Fig. 4a to the system, the optical and mechanical modes drastically change (Fig. 5b), destroying the transparency window of the OMIT (Fig. 5c, right).

In summary, we proposed to achieve opto-mechanically induced transparency by engineering the topology of an opto-mechanical crystal, made from a silicon thin wafer perforated with an array of rectangular holes. Due to its topological nature, the achieved OMIT spectral lineshape was not sensitive to geometrical tolerances, potentially enabling a large variety of novel applications such as interferometry, signal processing, and ultra-fast switching.

Funding. Swiss National Science Foundation, Grant number of 172487

Disclosures. The authors declare no conflicts of interest.

\section{References}

1. K. J. Boller, A. Imamoğlu, and S. E. Harris, Phys. Rev. Lett. 66, 2593 (1991)

2. M. Fleischhauer, A. Imamoglu, and J. P. Marangos, Rev. Mod. Phys. 77, 633 (2005).

3. J. J. Longdell, E. Fraval, M. J. Sellars, and N. B. Manson, Phys. Rev. Lett. 95, 063601 (2005).

4. M. O’Donnell, E. T. Jaynes, and J. G. Miller, J. Acoust. Soc. Am. 69, 696 (1981). 5. D. K. Hunter, M. C. Chia, and I. Andonovic, J. Light. Technol. 16, 2081 (1998). 6. Dorren, H. J. S., et al. J. Light. Technol. 21, 2 (2003).

7. A. I. Lvovsky, B. C. Sanders, and W. Tittel, Nat. Photonics, 3, 706 (2009).

8. R. M. Gingrich, P. Kok, H. Lee, F. Vatan, and J. P. Dowling, Phys. Rev. Lett. 91, 217901 (2003)

9. R. Ulrich. Opt. Lett, 5, 173 (1980).

10. K. Itoh, T. Saitoh, and Y. Ohtsuka, J. Light. Technol. 5, 916 (1987).

11. I. Fazal, O. Yilmaz, S. Nuccio, B. Zhang, A. E. Willner, C. Langrock, and M. M Fejer. Opt. Express, 15, 10492 (2007).

12. P. Blixt, J. E. Bowers, E. Bodtker, O. Sahlen, and R. S. Smith. IEEE Trans Microw Theory Tech, 43, 2214 (1995).

13. D. D. Smith, H. Chang, K. A. Fuller, A. T. Rosenberger, and R. W. Boyd. Phys. Rev. A. 69, 063804 (2004).

14. K. Totsuka, N. Kobayashi, and M. Tomita, Phys. Rev. Lett. 98, 213904 (2007)

15. M. Mancinelli, R. Guider, P. Bettotti, M. Masi, M. R. Vanacharla, and L. Pavesi, Opt. Express, 19, 12227 (2011).

16. Q. Xu, S. Sandhu, M. L. Povinelli, J. Shakya, S. Fan, and M. Lipson, Phys. Rev. Lett. 96, 123901 (2006).

17. N. Liu, L. Langguth, T. Weiss, J. Kästel, M. Fleischhauer, T. Pfau, and H. Giessen, Nat. Mater, 8, 758 (2009).

18. M. F. Limonov, M. V. Rybin, A. N. Poddubny, and Y. S. Kivshar, Nat. Photonics, 11, 543 (2017).

19. C. Wu, A. B. Khanikaev, R. Adato, N. Arju, A. A Yanik., H. Altug, and G. Shvets, Nat. Mater. 11, 69 (2012).

20. Q. Liao, X. Xiao, W. Nie, and N. Zhou, Opt. Express, 28, 5288-5305 (2020)

21. H. Xiong, and Y. Wu, Appl. Phys. Rev., 5, 031305 (2018).

22. A. H. Safavi-Naeini, et al. Nature. 472, 69 (2011).

23. M. Eichenfield, J. Chan, R. M. Camacho, K. J. Vahala, and O. Painter, Nature. 462, 78 (2009).

24. F. Zangeneh-Nejad, and R. Fleury. Nat. Comm. 10, 1 (2019).

25. L. Lu, J. D. Joannopoulos, and M. Soljačić, Nat. Phot. 8, 821 (2014).

26. F. Zangeneh-Nejad, and R. Fleury. Phys. Rev. Lett. 125, 054301 (2020).

27. F. Zangeneh-Nejad, and R. Fleury. Phys. Rev. Lett. 123, 053902 (2019).

28. F. Zangeneh-Nejad, and R. Fleury. Adv. Mater., 2001034 (2020).

29. M. Hafezi, E. A. Demler, M. D. Lukin, and J. M. Taylor, Nat. Phys. 7, 907. (2011).

30. X. Cheng, C. Jouvaud, X. Ni, S. H. Mousavi, A. Z. Genack, and A. B. Khanikaev, Nat. Mater, 15, 542 (2016).

31. C. He, et al. Nat. Phys. 12, 1124-1129 (2016).

32. A. B. Khanikaev, R. Fleury, S. H. Mousavi, and A. Alu, Nat. Comm. 6, 1-7 (2015).

33. S. D. Huber, Nat. Phys. 12, 621 (2016).

34. L. M. Nash, D. Kleckner, A. Read, V. Vitelli, A. M. Turne, and W. T. Irvine, PNAS. 112, 14495 (2015).

35. F. Zangeneh-Nejad, and R. Fleury, Phys. Rev. Lett. 122, 014301 (2019)

\section{References}


1. K. J. Boller, A. Imamoğlu, and S. E. Harris, "Observation of electromagnetically induced transparency." Phys. Rev. Lett. 66, 2593 (1991)

2. M. Fleischhauer, A. Imamoglu, and J. P. Marangos, "Electromagnetically induced transparency: Optics in coherent media." Rev. Mod. Phys. 77, 633 (2005).

3. J. J. Longdell, E. Fraval, M. J. Sellars, and N. B. Manson, "Stopped light with storage times greater than one second using electromagnetically induced transparency in a solid." Phys. Rev. Lett. 95, 063601 (2005).

4. M. O’Donnell, E. T. Jaynes, and J. G. Miller, "Kramers-Kronig relationship between ultrasonic attenuation and phase velocity." J. Acoust. Soc. Am. 69, 696 (1981).

5. D. K. Hunter, M. C. Chia, and I. Andonovic, "Buffering in optical packet switches." J. Light. Technol. 16, 2081 (1998).

6. Dorren, H. J. S., et al. J. "Optical packet switching and buffering by using alloptical signal processing methods." Light. Technol. 21, 2 (2003).

7. A. I. Lvovsky, B. C. Sanders, and W. Tittel, "Optical quantum memory." Nat. Photonics, 3, 706 (2009).

8. R. M. Gingrich, P. Kok, H. Lee, F. Vatan, and J. P. Dowling, "All linear optical quantum memory based on quantum error correction." Phys. Rev. Lett. 91, 217901 (2003)

9. R. Ulrich. "Bending-induced birefringence in single-mode fibers." Opt. Lett, 5, 173 (1980).

10. K. Itoh, T. Saitoh, and Y. Ohtsuka, "Optical rotation sensing by the geometric effect of fiber-loop twisting." J. Light. Technol. 5, 916 (1987).

11. I. Fazal, O. Yilmaz, S. Nuccio, B. Zhang, A. E. Willner, C. Langrock, and M. M Fejer. "Optical data packet synchronization and multiplexing using a tunable optical delay based on wavelength conversion and inter-channel chromatic dispersion." Opt. Express, 15, 10492 (2007)

12. P. Blixt, J. E. Bowers, E. Bodtker, O. Sahlen, and R. S. Smith. "Optical data synchronization using tunable transmitters and nonzero dispersion links." IEEE Trans Microw Theory Tech, 43, 2214 (1995).

13. D. D. Smith, H. Chang, K. A. Fuller, A. T. Rosenberger, and R. W. Boyd. "Coupled-resonator-induced transparency." Phys. Rev. A. 69, 063804 (2004).

14. K. Totsuka, N. Kobayashi, and M. Tomita, "Slow light in coupled-resonatorinduced transparency." Phys. Rev. Lett. 98, 213904 (2007)

15. M. Mancinelli, R. Guider, P. Bettotti, M. Masi, M. R. Vanacharla, and L. Pavesi, "Coupled-resonator-induced-transparency concept for wavelength routing applications."Opt. Express, 19, 12227 (2011).

16. Q. Xu, S. Sandhu, M. L. Povinelli, J. Shakya, S. Fan, and M. Lipson, "Experimental realization of an on-chip all-optical analogue to electromagnetically induced transparency." Phys. Rev. Lett. 96, 123901 (2006)

17. N. Liu, L. Langguth, T. Weiss, J. Kästel, M. Fleischhauer, T. Pfau, and H. Giessen, "Plasmonic analogue of electromagnetically induced transparency at the Drude damping limit." Nat. Mater, 8, 758 (2009).

18. M. F. Limonov, M. V. Rybin, A. N. Poddubny, and Y. S. Kivshar, "Fano resonances in photonics." Nat. Photonics, 11, 543 (2017).

19. C. Wu, A. B. Khanikaev, R. Adato, N. Arju, A. A Yanik., H. Altug, and G. Shvets, "Fano-resonant asymmetric metamaterials for ultrasensitive spectroscopy and identification of molecular monolayers." Nat. Mater. 11, 69 (2012).

20. Q. Liao, X. Xiao, W. Nie, and N. Zhou, "Transparency and tunable slow-fast light in a hybrid cavity optomechanical system." Opt. Express, 28, 52885305 (2020)

21. H. Xiong, and Y. Wu, "Fundamentals and applications of optomechanically induced transparency", Appl. Phys. Rev., 5, 031305 (2018).

22. A. H. Safavi-Naeini, et al. "Electromagnetically induced transparency and slow light with optomechanics." Nature. 472, 69 (2011).

23. M. Eichenfield, J. Chan, R. M. Camacho, K. J. Vahala, and O. Painter, "Optomechanical crystals." Nature. 462, 78 (2009).

24. F. Zangeneh-Nejad, and R. Fleury. Topological analog signal processing. Nat. Comm. 10, 1 (2019)

25. L. Lu, J. D. Joannopoulos, and M. Soljačić, "Topological photonics." Nat. Phot. 8, 821 (2014)
26. F. Zangeneh-Nejad, and R. Fleury. Phys. Rev. Lett. 125, "Zero index Weyl metamaterials" 054301 (2020).

27. F. Zangeneh-Nejad, and R. Fleury. "Nonlinear Second-Order Topological Insulators." Phys. Rev. Lett. 123, 053902 (2019).

28. F. Zangeneh-Nejad, and R. Fleury. Adv. Mater., "Disorder-Induced Signal Filtering with Topological Metamaterials" 2001034 (2020).

29. M. Hafezi, E. A. Demler, M. D. Lukin, and J. M. Taylor, "Robust optical delay lines with topological protection." Nat. Phys. 7, 907 (2011).

30. X. Cheng, C. Jouvaud, X. Ni, S. H. Mousavi, A. Z. Genack, and A. B. Khanikaev, "Robust reconfigurable electromagnetic pathways within a photonic topological insulator." Nat. Mater, 15, 542 (2016).

31. C. He, et al. "Acoustic topological insulator and robust one-way sound transport." Nat. Phys. 12, 1124-1129 (2016).

32. A. B. Khanikaev, R. Fleury, S. H. Mousavi, and A. Alu, "Topologically robust sound propagation in an angular- momentum-biased graphene-like resonator lattice," Nat. Comm. 6, 1-7 (2015).

33. S. D. Huber, "Topological mechanics." Nat. Phys. 12, 621 (2016).

34. L. M. Nash, D. Kleckner, A. Read, V. Vitelli, A. M. Turne, and W. T. Irvine, "Topological mechanics of gyroscopic metamaterials."PNAS. 112, 14495 (2015)

35. F. Zangeneh-Nejad, and R. Fleury, "Topological fano resonances." Phys. Rev. Lett. 122, 014301 (2019) 